\title{
Th Thema \\ Diversité et égalité en éducation (2000-2019) : évolutions, enjeux et perspectives
}

Zoe Moody, Haute école pédagogique du Valais et Centre interfacultaire en droits de l'enfant, Université de Genève

Isabel Valarino, Service de la recherche en éducation (SRED), Département de l'Instruction publique de la Formation et de la Jeunesse, Canton de Genève

Face à la diversité des publics de l'éducation, la thématique de l'égalité constitue un objet d'attention dans les recherches en éducation. La persistance des inégalités et le difficile équilibre à trouver entre prise en compte des besoins particuliers et non-discrimination sont au cour des débats, pour tenter de mettre en lumière les défis théoriques et pratiques de la diversité dans l'éducation. Cette contribution propose une revue systématique du champ de recherche traitant des questions de diversité et d'égalité en éducation, tel quill se dessine dans la principale revue du domaine des sciences de l'éducation en Suisse, durant les vingt dernières années (2000-2019). Les évolutions, points de rupture et continuités sont mis en lumière de même que les thématiques transversales les plus prégnantes, à partir de la description des enjeux sur lesquels travaillent les chercheuses et chercheurs en sciences de l'éducation.

\section{Introduction}

Face à la diversité des publics de l'éducation, la thématique de l'égalité ou partant de l'autre versant de la problématique, la question des inégalités, constitue un objet d'attention dans les recherches en éducation. ${ }^{1}$ Le difficile équilibre à trouver entre prise en compte de la différence et non-discrimination est au cœur des débats, pour tenter de mettre en lumière les défis théoriques et pratiques de l'hétérogénéité dans l'éducation. Qu'il s'agisse des dimensions influençant une idéale égalité de traitement - telles que le genre, l'origine ethnique ou socioéconomique ou encore des besoins éducatifs particuliers - ou des aspects permettant de comprendre et décrire la manière dont cette influence se joue ou peut être plus ou moins marquée (politiques, systèmes de formation, etc.), les manières d'aborder la problématique sont multiples.

La présente contribution s'ancre dans le cadre d'un appel spécial visant à réaliser une rétrospective sur les publications parues dans la Revue Suisse des sciences de l'Éducation (RSSE). Elle propose ainsi une revue systématique du champ de recherche traitant des questions de diversité et d'égalité en éducation, tel qu'il se dessine dans la principale revue du domaine des sciences de l'éducation en Suisse, notamment reconnue pour son caractère multilingue et multiculturel, durant les vingt dernières années (2000-2019). Cette revue systématique vise, d'une part, à mettre en lumière les évolutions, points de rupture et continuités dans le champ afin d'offrir une lecture de ces questions ancrée dans la temporalité de ces deux décennies. D’autre part, elle ambitionne d'identifier les thématiques transversales les plus prégnantes, à partir de la description des enjeux sur lesquels travaillent les chercheuses et chercheurs en sciences de l'éducation, au-delà de la thématique ou de l'approche spécifique.

Après avoir clarifié les enjeux méthodologiques et objectifs de cette contribution, une analyse organisée par dimensions influençant l'égalité en éducation est proposée. Sur la base du corpus des articles publiés dans la revue, ce sont les dimensions de genre, d'origine ethnique/parcours migratoire, ou socio-économique ou encore de besoins éducatifs particuliers qui ont été retenues. Dans un deuxième temps, les questions des méthodes et des terrains représentés dans le corpus sont analysées. Finalement, une discussion de ces résultats est proposée.

1 Nous tenons ici à remercier tout particulièrement les experte et expert, Isabelle Collet et Alain Muller pour leurs retours détaillés et constructifs, qui nous ont grandement servi pour la finalisation de cet article. 


\section{Méthodologie et objectifs}

Une recherche qui vise à établir des connaissances nouvelles sur la base de recherches existantes peut être conceptualisée et conséquemment menée de différentes manières. Méta-analyse est le terme généralement utilisé pour décrire la combinaison et articulation d'études quantitatives avec pour objectif de renforcer des éléments de preuve ou de proposer une interprétation plus globale des résultats. Une telle approche repose sur certaines procédures spécifiques, par exemple une évaluation rigoureuse de la qualité des recherches incluses dans l'analyse et l'utilisation des stratégies de recherche qui garantissent que la totalité des études portant sur l'objet soit trouvée. Lorsque ce sont les résultats d'études qualitatives qui sont résumés pour construire une base solide d'actions concrètes ou de recherches complémentaires, c'est le terme de revue systématique qui est appliqué. Une revue systématique est moins stricte qu'une méta-analyse quant au respect de certaines procédures ; dans un tel cas, il n'y a, par exemple, pas d'évaluation spécifique de qualité outre celui de l'examen par les pair.e.s pour publication, lequel est jugé adéquat pour l'analyse d'un échantillon représentatif de la littérature existante. Une troisième conceptualisation est la synthèse de recherches, laquelle n'a pas pour ambition d'établir des preuves ou de synthétiser des résultats, mais d'identifier des caractéristiques, des modèles voire les modes d'organisation des connaissances au sujet d'un objet donné et ainsi créer des connaissances qui vont au-delà de la simple addition des connaissances existantes à ce sujet (Andrews et Harlen, 2006 ; Quennerstedt, 2011 ; Quennerstedt et Moody, 2020 ; Soilemezi et Linceviciute, 2018 ; Sutcliffe et al., 2012).

L'approche favorisée dans le cadre de cet article combine certaines caractéristiques d'une revue systématique et d'une synthèse de recherche, nous permettant de réaliser ce que nous appellerons une revue systématique d'un champ de recherche, tel qu'il se dessine dans une revue multilingue et multiculturelle durant une période donnée. Il ne s'agit donc ni d'une méta-analyse ni d'une revue systématique au sens où nous ne visons pas à établir des preuves ou à résumer des conclusions. Notre étude porte davantage sur la description, l'organisation et l'interprétation de la combinaison de toutes les recherches publiées dans une revue spécialisée en sciences de l'éducation sur la question de la diversité en éducation, sans mettre l'accent sur les résultats, mais en cherchant à identifier des thèmes ou des méthodes de recherche privilégiés, caractéristiques de la manière dont la problématique est abordée. Une attention est prêtée aux résultats au même titre qu'à d'autres caractéristiques (thème, focale, méthode, terrain d'investigation) permettant de décrire, comme l'ambitionne la Revue Suisse des sciences de l'Éducation (RSSE), le " débat scientifique " sur ces questions ainsi que "les principales tendances de la recherche dans les différentes régions linguistiques de Suisse mais aussi d'ailleurs ».

Une recherche systématique des publications parues dans la RSSE a été effectuée à l'aide du moteur de recherche de la revue. Cette recherche a été réalisée en français, dans la mesure où tous les articles de la RSSE comportent une traduction en quatre langues (français, anglais, allemand et italien) du titre, du résumé et des mots-clés. Seuls les articles évalués par des pair-e.s, publiés entre 1999 et 2019 et correspondant aux termes de recherche présentés ci-dessous, dans le titre, le résumé ou les mots-clés ont été retenus pour une analyse détaillée. Les éditoriaux et recensions ne comportant ni résumé ni mots-clés ont par conséquent été exclus de notre étude.

Liste des mots-clés utilisés :

1. Égalité, inégalité(s)

2. Équité

3. Discrimination

4. Diversité

5. Hétérogénéité

6. Pluralisme

7. Genre (les articles portant sur le "genre" de texte ont été exclus)

8. Sexe (les articles abordant cette question sous un angle autre qu'une variable statistique ont été exclus)

9. Homosexualité

10.LGBT(QI)

11. Migration

12. Multiculturel, interculturel

13. Ethnicité (ethnique)

14. Classe sociale

15. Besoins éducatifs particuliers (BEP)

16. Handicap

17. Inclusion

18. Intersectionnalité (intersectionnel) 
Le fait que cet échantillon soit saturé à la fin du processus laisse penser que les principaux articles de la Revue portant explicitement sur les questions de diversité et d'égalité ont été identifiés. Nous reconnaissons que des oublis restent possibles bien que la marge d'erreur paraisse faible.

Un contrôle du fait que les articles traitent centralement ou partiellement de questions de diversité et d'égalité a été réalisé en amont de l'analyse. Les publications finalement retenues $(\mathrm{N}=65)$ ont ensuite été étudiées au travers de la lecture de leurs résumés, l'objectif étant d'identifier les descripteurs de la diversité et les dimensions des inégalités abordées, les méthodes de recherche ainsi que les contextes géographiques, nationaux ou régionaux dans lesquels s'ancrent les travaux.

Après cette première analyse, nous avons, d'une part, mis en évidence les évolutions, points de rupture éventuels et continuités afin de pouvoir offrir une lecture de ces questions ancrée dans la temporalité de ces deux décennies. D'autre part, nous avons identifié les thématiques transversales les plus prégnantes, permettant de décrire les enjeux sur lesquels travaillent les chercheuses et chercheurs en sciences de l'éducation, au-delà de la thématique plus spécifique.

\section{Analyse}

L'analyse du corpus d'articles retenus permet de mettre en évidence le fait que plusieurs angles d'approche sont mobilisés par les chercheuses et chercheurs en sciences de l'éducation lorsqu'il s'agit d'étudier les questions de la diversité et de l'égalité. Un point d'entrée est celui des dimensions descriptives de la diversité - genre, origine migratoire ou ethnique, classe sociale et besoins éducatifs particuliers ${ }^{2}$ - et mettant au défi les questions d'égalité. Lensemble des articles couvrent au moins une de ces dimensions, plus rarement plusieurs, c'est la raison pour laquelle nous utilisons ci-après cette organisation pour décrire les recherches publiées au sein de la RSSE durant les deux dernières décennies, et comment ces dimensions sont mises en lien avec d'autres thématiques de recherche (politiques d'égalité, système de formation, curriculum, évaluation, orientation scolaire, etc.).

Une première analyse chiffrée de l'évolution des publications en fonction des dimensions descriptives de la diversité indique que la plupart des travaux publiés portent sur les questions de genre (27), d'origine migratoire (25) et de classe sociale (18) (voir Graphique 1). Les travaux portant sur les élèves à besoins éducatifs particuliers (BEP) demeurent plus rares (9) bien qu'ils aient augmenté de manière marquée dans le courant de la dernière décennie. Les variations entre les deux périodes identifiées dans le cadre de cette recherche (2000-2009 et 2010-2019) ne permettent pas d'identifier de réelle rupture ou développement pour les trois premières dimensions. La diminution de publications en lien avec le genre s'explique par la parution de deux numéros spéciaux sur la question entre 2000 et 2009. En ce qui concerne la question des élèves à BEP, l'évolution entre une publication durant la première décennie et huit durant la seconde semble refléter la chronologie du développement des politiques éducatives et de l'école inclusive.

\section{a. Genre}

Les articles développant une réflexion abordant la question du genre comme variable explicative d'inégalités de chances ou de traitement, voire d'exposition à des violences ou du harcèlement, en Suisse ou ailleurs, ont été catégorisés comme relevant d'une approche " genre " de la diversité et de l'égalité. Cette approche est celle qui a été la plus largement mobilisée dans le cadre des travaux publiés dans la RSSE autour de ces questions (Figure 1). Deux numéros thématiques - Genre et formation professionnelle (2/2000) et Les relations de genre un contexte de transformation de la politique éducative et institutionnelle (2/2007) - permettent d'expliquer cette forte représentation au sein de notre corpus ainsi que les thématiques liées à cette dimension, telles que nous les présentons ci-après.

2 Bien que les besoins éducatifs particuliers, ou plus spécifiquement le handicap, soient une forme de diversité, celle-ci n'est pas comparable en tous points aux dimensions de "genre, race, classe ", qui sont des constructions sociales exclusivement. La complexité de la question du handicap incite à prévoir une variété d'approches et pistes d'intervention pour considérer l'articulation de ses aspects médicaux, éducatifs et sociaux (Winzer et Mazurek, 2017). Dans le cadre d'une revue systématique d'un champ de recherche, il convient toutefois d'opter pour des approches inclusives des thématiques, parfois en marge du champ, pour en saisir l'ampleur et les points de rencontre. Dans ce cas précis, la considération d'un droit à l'égalité en éducation invite à considérer les articles traitant des besoins éducatifs particuliers également (voir notamment Cole, 2018; Bennett Woodhouse, 2020) comme l'a d'ailleurs fait un des articles inclut dans cette étude : Gremion et al. (2013). 
Figure 1

Nombre d'articles par dimension étudiée (par période)

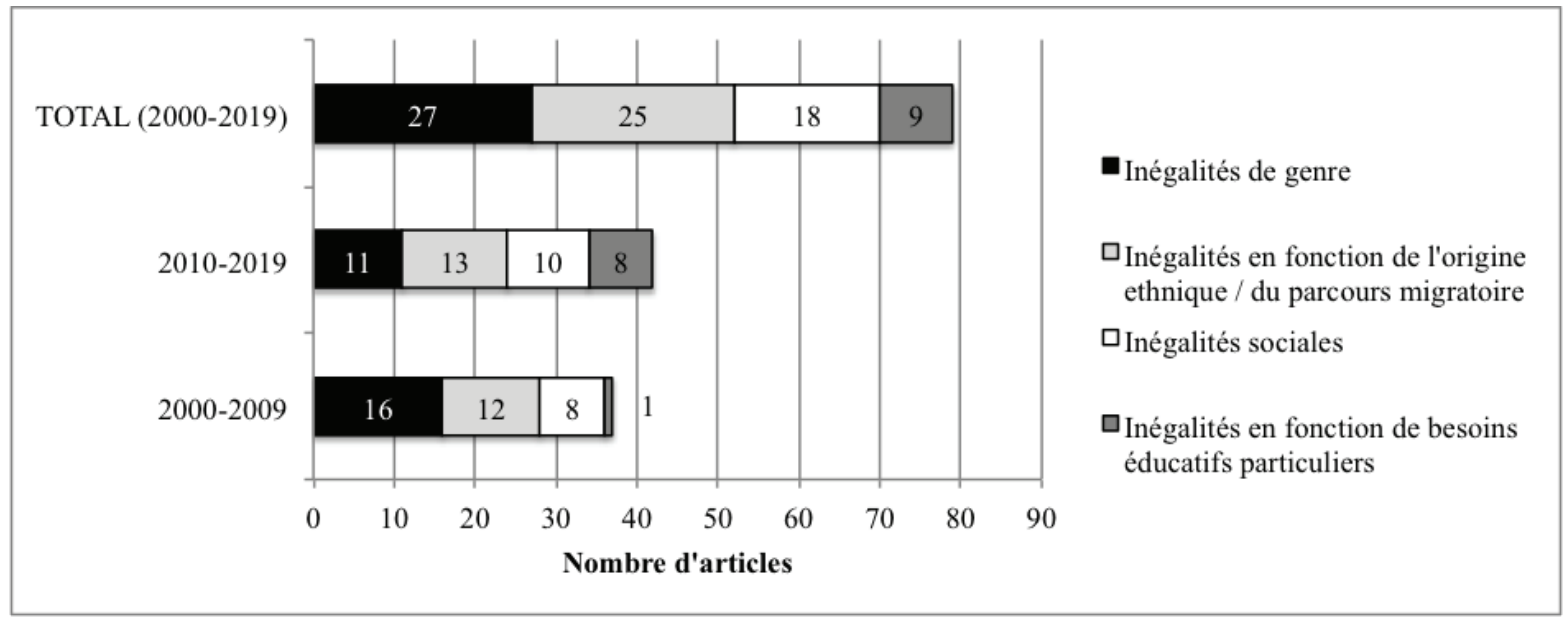

De manière marquée, les questions d'orientation scolaire ou professionnelle sont le plus fréquemment abordées. Bien qu'un numéro thématique portant explicitement sur la formation professionnelle soit un facteur explicatif, la présence-même d'un tel numéro reflète une attention particulière pour la question et l'existence de travaux de recherche conduits en Suisse et à l'international. Les travaux en question s'articulent autour de trois axes d'investigation principaux: (a) la segmentation/stratification des systèmes de professions et conséquemment d'orientation et de formation professionnelles, (b) les discriminations directes et indirectes auxquelles sont confrontés les individus des deux sexes durant leur formation et au travers des systèmes de formation et (c) les obstacles auxquels font face les femmes dans leur progression professionnelle ou carrières. Sur l'ensemble de la période, il convient de souligner l'absence totale d'articles traitant majoritairement de dimensions constitutives de la question "genre ", à savoir les questions d'orientation sexuelle et d'identité de genre. Les mots-clés homosexualité, LGBT(QI), intersectionnalité, n’ont en effet donné aucun résultat : ce qui laisse voir une tache aveugle de la recherche publiée dans la RSSE sur ces objets.

Les chercheuses et chercheurs travaillant sur les questions de segmentation ou stratification des systèmes de profession les renvoient aux rapports sociaux de sexe plus largement, lesquels sont structurés comme un système de division et de domination entre les sexes (Mosconi, 2000 ; Guidici et Manz, 2018). Conséquemment, ils et elles observent que l'orientation et la formation professionnelle, soit la profession et l'apprentissage en entreprise, désavantagent les femmes en Suisse (Borkowsky, 2000) et ailleurs (Heikkinen, 2000). Des études montrent d'ailleurs que les jeunes Suissesses rencontrent plus de difficultés dans la recherche d'une place d'apprentissage après l'école que leurs homologues masculins (Hirschi, 2009). Cette segmentation entre garçons et filles est aussi observée au niveau des cursus d'études ou des systèmes de formation : les études littéraires et tertiaires pour les filles et scientifiques et techniques industrielles pour les garçons ou, dans une perspective historique, les cours ménagers destinés aux femmes (Schärer, 2000). Une étude conduite au Luxembourg identifie la segmentation comme un facteur de risque pour les garçons, plus exposés au décrochage scolaire (Backes et Hadjar, 2017).

Les choix de cursus en mathématiques, en informatique, en sciences naturelles ou en technique (MINT) ou les attitudes socioaffectives des filles et des garçons face aux mathématiques font l'objet de plusieurs recherches (Aeschlimann et al., 2015 ; Genoud et al., 2015 ; Labudde, 2010). Un article étudie l'intérêt porté par des lycéennes et lycéens au métier d'enseignant - considéré comme offrant un bon salaire et la possibilité d'exercer un emploi à temps partiel - et les facteurs qui influencent la décision de suivre une formation pédagogique (Berweger et al., 2015).

Les discriminations auxquelles les jeunes filles et femmes sont confrontées durant leurs carrières scolaires et professionnelles sont au cœur de plusieurs publications. Celles-ci mettent en lumière que ces discriminations se jouent tout au long du parcours des jeunes et pas uniquement au moment de l'orientation ou de la formation professionnelle (formation de base et continue, réintégration professionnelle, etc.). Elles soulignent d'ailleurs la faible efficacité des mesures ciblant l'information et la motivation des jeunes femmes en insistant sur l'importance d'engager des changements structurels (Grossenbacher, 2000 ; Unwin, 2000 ; voir aussi Fassa et Naef, 2015). 
Une attention particulière est aussi portée à l'inégalité des femmes dans la progression professionnelle ou au cours de leur carrière scientifique. Ces recherches indiquent que si les femmes entrent dans la vie professionnelle avec un statut supérieur, leur mobilité verticale est inférieure, ce qui est lié à une répartition " traditionnelle " des rôles sociaux et familiaux (Häfeli et al., 2015). Dans l'académie, la disparité entre les femmes et les hommes occupant des postes à responsabilité ou professoraux ne s'explique pas par la sous-représentation des femmes aux échelons inférieurs de la carrière scientifique. Au contraire, ces travaux invitent à remettre en cause des hypothèses répandues à ce sujet et à porter une attention particulière aux disciplines au sein desquelles l'égalité des chances se joue de manière sensiblement différente (Leemann, 2002 ; Lind et Löther, 2007).

\section{b. Origine migratoire et ethnique}

Un nombre significatif de publications du corpus étudié abordent la diversité éducative du point de vue de l'origine migratoire et/ou ethnique des élèves. De manière intéressante, aucun numéro spécial sur cette question n'est paru - bien que deux numéros publiés à dix ans d'écart, l'un sur les contextes transnationaux et l'autre sur les frontières éducatives, aient généré la parution de plusieurs articles portant sur la thématique - et le nombre d'articles sur les périodes étudiées n'a pas varié. Ces travaux s'organisent autour de trois axes principaux. Premièrement, ils questionnent les politiques et systèmes éducatifs et leur capacité à prendre en compte la diversité ethnique et migratoire. Deuxièmement, les approches pédagogiques spécifiques pour prendre en compte l'hétérogénéité des publics sont analysées. Finalement, les recherches abordent les rôles des enseignant.e.s ainsi que de la famille.

Se focalisant sur la question des politiques éducatives et les systèmes de formation, plusieurs autrices et auteurs mettent en évidence une prise en compte "timide " de la pluralité et de l'hétérogénéité des contextes éducatifs à ces différents niveaux. Étudiant le statut de la pluralité dans différents contextes sociopolitiques (ici la France et le Québec), une contribution met en lumière la tension entre la pluralité culturelle comme " problème appelant un traitement » et le pluralisme comme valeur. D'autres travaux comparatifs sur les mêmes contextes ou d'autres radicalement différents (État national-socialiste et République démocratique allemande) montrent comment le cadre législatif peut être plus ou moins respectueux des droits des enfants, migrant.e.s ou d'origine étrangère, en particulier du droit à l'éducation (Ruşitoru, 2017 ; Tenorth, 2008).

Plusieurs articles soulignent le traitement inégal auquel font face les minorités ethniques ou linguistiques au cœur des systèmes de formation et la persistance de ces inégalités (Bakes et Hadjar, 2017 ; Müller, 2001 ; Unwin, 2000), y compris face à la violence entre élèves (Oertel et al., 2009). À l'inverse, une recherche comparative France, Suisse et Allemagne indique que certains groupes de jeunes d'origine migrante et socialement défavorisés accèdent davantage aux études supérieures que leurs homologues nationaux (Griga et Hadjar, 2013). À noter que ces inégalités sont très fréquemment liées aux inégalités socio-économiques et à celles de genre dans une moindre mesure.

Plusieurs travaux parus dans le numéro portant sur les contextes transnationaux invitent à prendre en compte les avancées théoriques sur la migration et la pluralité pour développer de nouvelles recherches et approches interdisciplinaires. Celles-ci seraient en effet susceptibles de dépasser la seule considération du degré d'intégration ou de la différence pour entrer dans un paradigme de la diversité capable de reconnaitre les ressources et opportunités qu'elle recouvre en éducation. Ces autrices et auteurs en appellent à dépasser l'illusion d'homogénéité des systèmes et de la population scolaires (Akkari, 2007 ; Neumann, 2007 ; Perregaux, 2007 ; Prengel, 2007).

Concrètement, plusieurs contributions portent ainsi une attention particulière sur l'éducation interculturelle, multiculturelle, antiraciste et à la citoyenneté comme des approches spécifiques de l'hétérogénéité en contexte scolaire. Au cœur de ces articles se trouve la question de savoir comment apprendre de et par la diversité pour construire la cohésion sociale. D’une part, est soulignée la question de comment « concilier diversité des connaissances et savoirs communs, diversité de socialisations et culture commune " (Perregaux, 2007). D'autre part, l'indifférence de ces approches pédagogiques à la prise en compte des stades du développement des enfants et des adolescent.e.s comme apprenant.e.s est regrettée (Blakeney, 2002). Une contribution s'intéresse plus précisément aux liens entre ces approches et celle de la pédagogie spécialisée, dépassant ainsi l'habituelle et intentionnelle distanciation entre ces courants pour focaliser l'attention sur les convergences et divergences de leurs discours sur la différence et l'impact sur les pratiques (Gremion et al., 2013).

L'intégration de ces approches spécifiques dans la formation des enseignant.e.s fait également l'objet d'études, soulignant l'importance d'appréhender pleinement l'hétérogénéité d'un contexte scolaire encore trop perçu comme monoculturel (Leutwyler et al., 2009 ; Prengel, 2007). Une série de travaux portent également sur les pratiques des enseignant.e.s et directions pour réduire les inégalités et favoriser la réussite scolaire (voir Bauer, 2019). Un article remarque d'ailleurs que la littérature ne fournit pas de définition unique de la notion « issu.e 
de l'immigration » engendrant notamment une tendance chez les enseignant.e.s à sous-estimer la part des enfants d'origine immigrée dans leurs classes (Dubowy et al., 2011).

La question de la compétence linguistique des élèves allophones est aussi régulièrement thématisée dans la mesure où elle représente une condition essentielle de la réussite scolaire (voir Imgrund, 2017). L'enseignement des langues est ainsi problématisé en soulignant l'importance des pratiques enseignantes pour favoriser un développement des capacités langagières qui tienne compte des conditions familiales différentes (Kuyumcu et Senyldiz, 2011) et favorise la conservation de la langue d'origine et ses apports potentiels à la scolarisation (Sánchez Abchi, 2015). Finalement, quelle que soit la définition que l'on retienne des élèves issu.e.s de la migration ou bilingues, ces travaux révèlent que tous les critères sont en mesure de soutenir l'identification précoce des enfants présentant un risque élevé de déficit linguistique (Dubowy et al., 2011).

\section{c. Origine sociale}

La catégorie d'articles portant sur l'origine sociale comme descriptive de la diversité en contexte d'éducation et formation comprend une série de publications utilisant la variable de l'origine sociale, socio-économique ou de la classe sociale comme axes d'analyse. Ces publications - dont certaines ont été intégrées au numéro spécial paru en 2013 : Inégalité et justice dans l'éducation - portent principalement sur les questions d'orientation scolaire, de systèmes de formation, de politiques et des enseignant.e.s.

Les travaux conduits sur les systèmes de formation et l'orientation scolaire ou professionnelle mettent en évidence la corrélation entre la stratification des systèmes scolaires et les inégalités scolaires, questionnant alternativement la démocratisation des études, l'accès aux études supérieures ainsi que les effets de la perméabilité au sein des systèmes de formation. Ces travaux sont très hétérogènes quant aux terrains d'études; on trouve autant de travaux conduits à l'étranger qu'en Suisse.

Proposant des analyses de la diversité des situations éducatives et des contextes scolaires, plusieurs recherches se centrent sur l'accès aux études supérieures et l'inégalité des chances en la matière. Les liens entre ségrégation sociale de l'habitat et situations éducatives inégales sont questionnés par deux publications. L'une s'intéresse au contexte français et remet en cause une traduction de la ségrégation territoriale et de l'ancrage des établissements scolaires dans ces régions, soulignant le rôle actif de l'école qui participe à la définition de ces situations (Duru-Bellat, 2001). L'autre montre comment, en Belgique, la composition sociale des établissements scolaires influence significativement le choix d'aller à l'université ou non (Dupont et Lafontaine, 2011).

Les travaux conduits en Suisse indiquent également une forte corrélation entre l'origine sociale, le cursus d'études, le diplôme obtenu et les pratiques d'évaluation. Une étude fribourgeoise portant sur le passage de l'école primaire à l'école secondaire montre l'influence de l'origine des élèves sur la voie d'études à laquelle les élèves ont accès (Biewer et al., 2013). Analysant l'influence de l'origine sociale sur la probabilité d'accéder au gymnase/lycée après l'école obligatoire, chercheuses et chercheurs soulignent aussi les effets primaires et secondaires de l'origine sociale pour l'accès aux études supérieures (20\% d'après Combet, 2013).

Quelques travaux portent sur la perméabilité et les changements de voie d'études. Ils montrent que l'origine sociale influence le risque de réorientation scolaire et que les changements d'orientation lors des études au degré secondaire II diminuent la probabilité d'entrer à l'université. Ils concluent que la perméabilité contribue plutôt à reproduire les inégalités scolaires qu’à les réduire (Backes et Hadjar, 2017 ; Kost, 2013).

En matière de politiques de réduction des inégalités, des travaux proposent des regards sociohistoriques sur la démocratisation de l'enseignement en France, puis la centralisation et standardisation de l'éducation. Ils questionnent les effets réels de ces politiques, d'une part en montrant que l'inégalité entre les différentes filières évolue de façon variable et nuancée et d'autre part en relevant que l'autonomie des établissements présentée comme une promesse d'égalité traduit davantage un mode de management inspiré du capitalisme, reposant sur des normes internationales (Derouet, 2004 ; Prost, 2001).

L'influence des enseignant.e.s et directions d'établissement sur le climat scolaire et leur capacité à amoindrir les effets des inégalités sociales et œuvrer pour la réussite scolaire font l'objet de plusieurs publications. Celles-ci indiquent que les inégalités sociales influencent significativement le climat relationnel et organisationnel, voire le risque d'être victime de violences ou de harcèlement. Elles précisent qu'il est possible pour les enseignant.e.s ou les directions d'établissement de "faire une différence " pour améliorer le climat éducatif et favoriser l'établissement de relations coéducatives (Bauer, 2019; Lasne, 2017 ; Oertel et al., 2009 ; Quesel et al., 2014). Une publication thématise quant à elle, les liens entre les notions d'échec scolaire et d'accès aux savoirs, appelant à davantage de dialogue entre une sociologie des inégalités scolaires et la recherche en pédagogie et psychologie des apprentissages (Rochex, 2001). 


\section{d. Élèves à besoins éducatifs particuliers (BEP)}

La dernière catégorie d'articles de notre corpus regroupe ceux qui portent plus spécifiquement sur les besoins éducatifs particuliers. Neuf contributions en tout s'attaquent à cette forme de diversité et la quasi-totalité d'entre elles a été publiée durant la dernière décennie. Cette évolution traduit sans doute, comme susmentionné, une attention grandissante liée aux changements politiques en la matière. La plupart de ces contributions abordent d'ailleurs les questions politiques précisément, dans des contextes particuliers, et, de manière complémentaire, les dispositifs de soutien ainsi que le rôle des enseignant.e.s. Deux publications visent à proposer un état des lieux sous forme de synthèses de recherches.

Partant du constat que plusieurs pays et cantons suisses déploient des efforts dans le but de développer des systèmes scolaires plus inclusifs et d'intégrer si possible dans les classes ordinaires tous les élèves ayant des BEP, chercheuses et chercheurs ont proposé plusieurs articles se penchant sur le cadre, les effets et les pratiques de l'inclusion scolaire. La plupart des articles soulignent les avancements en la matière ainsi que les défis qui restent à relever. Au Tessin, depuis les années 1950, c'est une modification du rapport entre la société et l'école avec la démocratisation de l'accès aux études, puis une nouvelle vision des différences dans l'apprentissage, de l'inadaptation et de l'échec scolaires, qui sont observées (Dozio, 2001). À travers une approche historienne, une contribution met en évidence la rémanence de formes d'exclusion des élèves dont les comportements dérangent, à Fribourg, et ceci malgré le développement du principe d'intégration (Noël et al., 2019).

Quatre autres articles s'inscrivent dans le champ des recherches concernant les pratiques inclusives en milieu scolaire ordinaire. Une équipe examine, depuis l'adoption cantonale d'une politique d'intégration à Zürich, à quelles conditions les enseignant.e.s sont optimistes et pensent que la législation est mise en œuvre avec succès (Eckstein et al., 2013). Un article étudie l'évaluation formative comme moyen de prise en charge des élèves à BEP et comme source de progression collective (Barthélémy, 2015). Finalement, on trouve des travaux sur les défis de la transition entre l'école et la formation professionnelles des jeunes en situation de handicap en Suisse (Pool Maag, 2016) et la capacité perçue des enseignant.e.s en formation à gérer des comportements difficiles en classe (Bonvin et Gaudreau, 2015).

Deux contributions visent à dresser les contours des connaissances acquises dans ce champ de recherche émergeant. Une synthèse des recherches menées sur le développement de modèles théoriques de l'inclusion scolaire et sur l'évaluation des effets de différentes pratiques plus ou moins inclusives est ainsi réalisée (Rousseau et al., 2013). De même, une métasynthèse vise à produire une nouvelle compréhension des enjeux de l'intégration et de l'inclusion scolaires des élèves BEP (Rousseau et al., 2017).

\section{e. Données, méthodes et terrains d'investigation}

Parmi les articles pour lesquels les données et les méthodes d'analyse ont pu être déterminées ( $\mathrm{n}=52$ ), la plupart d'entre eux propose une analyse de type quantitative (31 articles), alors que 17 articles mobilisent des méthodes qualitatives et 4 articles proposent une revue de littérature.

Les types d'analyses quantitatives varient d'un article à l'autre. Plusieurs articles mobilisent des données représentatives de la population, souvent issues des statistiques officielles sur l'éducation, afin de décrire et de caractériser les inégalités étudiées. S’intéressant par exemple aux inégalités entre femmes et hommes à différents moments de leur parcours de formation, plusieurs contributions s'appuient sur des données chiffrées pour décrire et analyser des mécanismes de ségrégation (par exemple, Borkowsky, 2000 ; Mosconi, 2000 ; Stecher, 2007).

D'autres articles se basent sur des données issues de grandes enquêtes thématiques, telles que HBSC sur les comportements de santé et styles de vie des élèves, l'enquête TREE sur les transitions de l'école à l'emploi ou encore PISA. Ces études, ainsi que d'autres qui se basent sur des données recueillies pour répondre à des questions de recherche spécifiques, réalisent en général des analyses de type bivariées ou multivariées (analyses de variance et régressions). Ces analyses mettent en relation plusieurs variables entre elles et visent à étudier l'impact d'une ou de plusieurs d'entre elles sur un phénomène étudié. À titre d'exemple, une analyse de ce type permet de constater que l'origine sociale des élèves influence leur passage du primaire au secondaire, même après avoir contrôlé l'effet des recommandations des parents et des professeur.e.s (Biewer et al., 2013). Une autre équipe a étudié la manière dont l'identité culturelle d'élèves migrant·ess d'origine portugaise en Suisse varie en fonction de caractéristiques personnelles (sexe, date d'arrivée en Suisse), familiales (type de permis de travail de la mère) et scolaires (redoublement, niveau de réussite scolaire, fréquentation des cours de langue et de civilisation portugaises) (Pons et al., 2000).

Quelques articles mobilisent des analyses statistiques multiniveaux qui permettent de distinguer l'impact de variables individuelles et de contexte. Une étude belge montre par exemple que les projets d'études supérieures d'élèves du secondaire ne sont pas seulement dépendants de leurs caractéristiques individuelles mais également, 
dans certains cas, de l'établissement dans lequel ils et elles sont scolarisé.e.s (effet de la composition sociale de l'école) (Dupont et Lafontaine, 2011).

Les données et méthodes qualitatives sur lesquelles s'appuient bon nombre d'articles sont elles aussi variées. Les entretiens qualitatifs avec des enseignant.e-s, directions d'école ou autres actrices ou acteurs de l'institution scolaire constituent une source de données privilégiée. Ces outils sont parfois combinés avec des observations de type ethnographique.

D'autres travaux s'appuient sur l'analyse de différents types de documents, notamment d'archives sur les programmes scolaires, de matériel pédagogique sur l'égalité destiné au corps enseignant et de textes légaux. Enfin quelques articles plus récents (après 2015) proposent des analyses de cas - de jeunes, d'élèves, de dispositifs - qui visent à mettre au jour des mécanismes spécifiques, tels que les apprentissages informels dans la culture " en ligne " pour les jeunes dits "à risque " (Preite, 2018), l'impact des pratiques enseignantes pour l'enseignement du français langue étrangère à l'école primaire (Imgrund, 2017) ou encore l'évaluation d'un dispositif de co-éducation (Lasne, 2017).

On relèvera enfin quelques revues de la littérature ou synthèses de recherches, sur des sujets variés, allant de l'école inclusive (Rousseau et al., 2013, 2017) à la transnationalité (Prengel, 2007), en passant par des questions méthodologiques (Duru-Bellat, 2001).

En ce qui concerne les terrains d'investigation traités dans les articles du corpus, un peu plus de la moitié porte sur le contexte suisse uniquement (32 articles) et l'autre moitié concerne un ou plusieurs autres pays (27 articles). Les articles qui portent sur le contexte suisse traitent souvent de réalités cantonales spécifiques, ce qui est sans doute le reflet de la compétence cantonale et non fédérale de l'éducation et de la formation en Suisse. Parmi les 27 articles qui s'intéressent à un ou plusieurs autres pays que la Suisse, 18 articles se centrent sur un seul contexte national, la France et l'Allemagne étant les plus fréquents. Enfin, neuf articles comparent les situations dans plusieurs pays, parmi lesquels peut ou non figurer la Suisse. Ces travaux comparatifs visent à faire ressortir les différences caractérisant divers contextes culturels et éducatifs.

\section{Discussion et conclusion}

Trois points d'attention ressortent de cette revue systématique. Tout d'abord l'évolution des dimensions d'inégalités traitées dans les articles du corpus montre la tendance à une vision segmentée des facteurs producteurs d'inégalités dans les parcours scolaires et professionnels des élèves et non une approche intersectionnelle. Deuxièmement, l'analyse des thématiques traitées en lien avec les dimensions d'inégalités illustre certains points aveugles de la recherche sur la diversité et l'égalité. Troisièmement, les méthodes mobilisées par les chercheuses et chercheurs (qualitatives et quantitatives) sont peu souvent combinées entre elles et les recherches-actions sont quasi absentes. Peu d'articles permettent de dépasser les constats documentant les inégalités au sein du système scolaire.

On constate en effet que 65 articles de la RSSE ont traité de la diversité et de l'égalité au cours des 20 dernières années. Si l'on considère qu'environ 60 numéros ont été publiés dans cet intervalle, on peut conclure que ces thèmes demeurent relativement marginaux dans le champ des sciences de l'éducation. Par ailleurs, parmi les quatre dimensions d'inégalités retenues (de genre, de classe, d'origine migratoire et en fonction de besoins éducatifs particuliers), toutes n'ont pas reçu la même attention. Si d'un côté les inégalités de genre, de classe et en fonction de l'origine migratoire sont présentes de façon constante dans les articles publiés de 2000 à 2019, ce n'est pas le cas pour les inégalités liées aux besoins éducatifs particuliers des élèves qui ont principalement fait leur apparition au cours de la dernière décennie seulement (2010-2019).

Nous faisons l'hypothèse que ce décalage est lié à l'émergence relativement récente de ce champ de recherche, lui-même étant lié aux changements sociétaux et politiques de ces dernières décennies en faveur d'une école pour tou.te.s. Parmi ces changements on peut citer entre autres l'adoption de la déclaration de Salamanque formulant un cadre d'action pour l'éducation et les besoins spéciaux (UNESCO, 1994), la Convention relative aux droits des personnes handicapées (ONU, 2006), l'adoption de loi fédérale sur l'élimination des inégalités frappant les personnes handicapées en Suisse (LHand, Confédération Suisse, 2002) et l'Accord intercantonal sur la pédagogie spécialisée entré en vigueur en 2011 (CDIP, 2019). Dans ce contexte, il est possible que les travaux sur l'école inclusive, développés depuis les années 1990 principalement (Prud'Homme et al., 2016), soient surtout publiés dans des revues thématiques et soient encore peu représentés dans des revues plus généralistes telles que la RSSE. De plus, si les travaux mettant en avant le caractère construit et situé du handicap et des difficultés scolaires sont plus nombreux depuis quelques décennies, la vision médicale est toujours bien 
présente dans ce champ (Lavoie et al., 2013). Les questions LGBT(QI), qui sont les grandes absentes de ce corpus, dénotent une invisibilisation des aspects liés à l'orientation sexuelle et l'identité de genre en éducation comme objet d'étude à part entière dans les travaux publiés par la revue. Le fait que cette thématique soit émergente explique en partie cette absence dans la revue, bien que celle-ci nécessiterait toutefois une attention plus marquée.

À l'inverse, les travaux en sciences de l'éducation qui problématisent les inégalités de classe, de genre et en fonction de l'origine migratoire, se réferent à des champs de recherche en sciences sociales plus anciens. En effet, l'étude de la stratification et des classes sociales remonte notamment aux travaux d'Emile Durkheim et plus récemment s'inscrit dans la suite des travaux sur la reproduction sociale de Bourdieu et Passeron (1964), ainsi que Boudon (1973). Les études sur les femmes et le genre sont, quant à elles, marquées par plusieurs "vagues " successives depuis la fin du $19^{\mathrm{e}}$ siècle et elles imprègnent les sciences sociales de façon transversale (Parini, 2010 ; Pavard 2018). Enfin les travaux sur la migration, dont l'impulsion est partie des pays anglo-saxons, de manière plus récente certes, se sont multipliés en particulier depuis les années 1980-90 avec l'accélération de la globalisation et des approches transnationales (Levitt et Jaworsky, 2007 ; Mazzella, 2014).

Autre constat : la plupart des articles du corpus ne traitent que d'une seule dimension d'inégalité à la fois. En effet, seuls dix articles font référence dans leur résumé à l'analyse d'au moins deux dimensions parmi les quatre retenues. Lorsque c'est le cas, on peut constater que les travaux sont plutôt abordés, comme c'est le cas dans la recherche plus largement depuis un certain temps déjà, du point de vue du genre et de la classe sociale. ${ }^{3}$ Parfois, l'origine migratoire est incluse, mais jamais dans le sens inverse. Autrement dit, les dimensions du genre et de la classe sociale sont moins régulièrement mobilisées dans les travaux portant sur la migration. De plus, le lien entre les besoins éducatifs particuliers et le genre, la classe et la migration sont rarement thématisés, notamment en raison du caractère particulier des BEP comme forme de diversité (voir ci-dessus), mais aussi pour des raisons de « jeunesse " du champ sans doute.

Une autre limite est à relever quant aux quelques travaux qui intègrent plusieurs dimensions d'inégalités. La plupart d'entre eux traitent généralement ces dimensions indépendamment les unes des autres (par exemple, en analysant l'impact du genre sur un phénomène en contrôlant statistiquement l'influence de la classe sociale), et non comme des dimensions liées et en interaction les unes avec les autres. Or, c'est précisément ce que suggère l'approche intersectionnelle, qui vise à analyser la manière dont l'appartenance à différentes catégories sociales (par exemple de genre, de classe, de " race ») et leur articulation complexe ainsi que le positionnement des individus dans des rapports de pouvoir influencent leur(s) expériences(s) (Collins et Bilge, 2016). Il s'agit d'un outil qui permet de considérer la complexité de la réalité plutôt que de demeurer dans une approche analytique simple.

La tendance à intégrer une perspective intersectionnelle dans la recherche en éducation est désormais bien implantée dans les pays anglo-saxons (voir par exemple Mitchell et al., 2014 ; Eden, 2017 ; Gross et al., 2016). La recherche récente francophone et germanophone traduit également cette tendance, ${ }^{4}$ bien que celle-ci ne se reflète pas encore dans les travaux publiés dans une revue plus générale telle que la RSSE. À l'avenir, il semble pertinent que les travaux sur le genre et la diversité dans le domaine de l'éducation intègrent davantage la façon dont les différentes dimensions d'inégalités caractérisant les élèves sont liées et articulées entre elles, plutôt que de les considérer de façon isolée.

Le deuxième point d'attention porte sur les objets de recherche thématisés en lien avec l'égalité et la diversité. On constate que les recherches portent principalement sur la formation obligatoire et post obligatoire. Plusieurs recherches s'intéressent en particulier à la transition entre l'enseignement secondaire I et secondaire II ; un moment charnière d'orientation et de sélection des élèves en Suisse (Neuenschwander, 2007). S'il s'agit d'une transition comportant de forts enjeux en matière de parcours et d'inégalités, d'autres transitions tout aussi déterminantes sont, quant à elles, moins thématisées ; par exemple la période préscolaire et l'entrée en primaire, les passages entre l'enseignement régulier et l'enseignement spécialisé ou encore la transition de l'école à l'emploi.

On peut également remarquer que les travaux traitant du post obligatoire et du tertiaire le font majoritairement en analysant les effets du genre ou de la classe sociale. Il y a relativement peu d'articles sur les effets de la migration ou d'être un enfant à BEP à ces niveaux. Il y a également peu d'analyses du curriculum, donc peu

3 Parmi les travaux francophones en éducation ayant articulé le genre et la classe, on peut notamment citer Duru-Bellat (1989), Baudelot et Establet (1992) ainsi que Mosconi (1998).

4 Le colloque de Créteil en 2017 « Penser l'intersectionnalité dans les recherches en éducation », la " controverse " parue en 2019 (Belkacem et al.) ou encore l'ouvrage collectif de Kronberger (2017) en sont des traces tangibles. 
d'articles liés aux contenus d'apprentissage en tant que tels. À quelques exceptions près portant sur les questions de genre (Guidici et Manz, 2018 ; Labudde, 2010), une grande partie des études partent de cadres théoriques issus de la sociologie ou de la politique de l'éducation. D'ailleurs quelques articles du corpus lancent un appel à davantage d'interdisciplinarité (Akkari, 2007 ; Prengel, 2007).

Enfin, en ce qui concerne les méthodes d'investigation des articles traitant de l'égalité et de la diversité, une grande variété peut être notée, avec toutefois une dominante de l'approche quantitative. Cette variété reflète les divers moyens mis en œuvre par les chercheuses et chercheurs pour répondre à des objectifs et questions de recherche très différents. Il paraît essentiel de continuer à analyser les mécanismes et facteurs de reproduction des inégalités à l'échelle nationale, voire internationale, dans une perspective comparative et également de documenter et comprendre (notamment par des observations et/ou entretiens) aux niveaux meso- et microsocial ce qui se joue par exemple dans les interactions au sein des établissements, des classes, des familles et des relations familles-école.

À ce propos, notons la quasi-absence d'articles reposant sur des méthodes mixtes de recherche. Or, les méthodes mixtes peuvent être extrêmement riches, par exemple pour l'exploration de nouvelles hypothèses ou directions de recherches, ou encore pour la validation, la triangulation ou la généralisation de résultats. Elles sont par ailleurs un pont de choix entre méthodes quantitatives (prédominantes à l'heure actuelle) et qualitatives (Teddlie et Tashakkori, 2003). La recherche-action est elle aussi quasiment absente des articles du corpus. Pourtant, cette approche est souvent mobilisée en lien avec des objectifs de réforme et d'évaluation de politiques ou de mise en place de nouveaux dispositifs. Elle présente également la particularité d'impliquer les actrices et acteurs du système éducatif dans un processus de changement individuel ou sociétal (Pelt et Poncelet, 2011). De ce fait, de telles recherches pourraient apporter des pistes de réponses et de réflexions intéressantes pour aller au-delà du constat des inégalités et in fine contribuer à leur réduction.

\section{Références bibliographiques}

Aeschlimann, B., Herzog, W., et Makarova, E. (2015). Préférences d'études de lycéens et lycéennes : qui décide de suivre un cursus MINT et pour quelles raisons ? Revue Suisse des Sciences de l'Éducation, 37(2), 285-300. https://doi.org/10.24452/ sjer.37.2.4954

Akkari, A. (2007). Les impasses de l'école multiculturelle et de l'école républicaine dans une perspective comparative. Revue Suisse des Sciences de l'Éducation, 29(3), 379-398. https://doi.org/10.24452/sjer.29.3.4577

Andrews, R., et Harlen, W. (2006). Issues in synthesizing research in education. Educational Research, 48(3), 287-299.

Backes, S., et Hadjar, A. (2017). Trajectoires scolaires au sein de l'école secondaire du Luxembourg : les effets de la perméabilité sur les inégalités de formation. Revue Suisse des Sciences de l'Éducation, 39(3), 437-460. https://doi.org/10.24452/sjer.39.3.5040

Barthélémy, V. (2015). L'inclusion scolaire dans l'enseignement primaire : L'évaluative formative comme source de progression collective ? Revue Suisse des Sciences de l'Éducation, 36(3), 523-544. https://doi.org/10.24452/sjer.36.3.5110

Baudelot, C., et Establet, R. (1992). Allez les filles! Seuil.

Bauer, S. (2019). Diriger un établissement multiculturel et défavorisé : un défi à part ? Revue Suisse Des Sciences De l'éducation, 41(1), 177-193. https://doi.org/10.24452/sjer.41.1.12

Belkacem, L., Gallot, F., et Mosconi, N. (2019). Penser l'intersectionnalité dans le système scolaire ? Travail, Genre et Sociétés, 41(1), 147-152.

Bennett Woodhouse, B. (2020). Reimagining equality through the lens of human rights: Lessons from the United Nations Convention on the rights of the child. Fordham Urb. L.J., 353. https://ir.lawnet.fordham.edu/ulj/vol47/iss2/5

Berweger, S., Kappeler, C., Keck Frei, A., et Bieri Buschor, C. (2015). Plans de carrières atypiques - Quel est l'intérêt des gymnasiens pour le métier d'enseignant? Revue Suisse des Sciences de l'Éducation, 37(2), 321-340. https://doi.org/10.24452/sjer.37.2.4956

Biewer, C., Wandeler, C., et Baeriswyl, F. (2013). Les effets de l'origine sociale et les perceptions de justice pendant la transition de l'école primaire à l'école secondaire. Revue Suisse des Sciences de l'Éducation, 35(3), 425-446. https://doi.org/10.24452/ sjer.35.3.4918

Blakeney, R. (2002). Comment savoir si cela une chose de valeur : une approche développementale de l'éducation interculturelle et antiraciste. Revue Suisse des Sciences de l'Éducation, 24(3), 467-486. https://doi.org/10.24452/sjer.24.3.4640

Bonvin, P., et Gaudreau, N. (2015). Prévenir et gérer les problèmes de comportement en classe : préparation perçue de futurs enseignants suisses et québécois. Revue Suisse Des Sciences De l'éducation, 37(3), 481-502. https://doi.org/10.24452/sjer.37.3.4964

Borkowsky, A. (2000). Les femmes et les hommes dans la formation professionnelle en Suisse. Revue Suisse des Sciences de l'Éducation, 22(2), 279-294. https://doi.org/10.24452/sjer.22.2.4567

Boudon, R. (1973). L’inégalité des chances. La mobilité sociale dans les sociétés industrielles. Armand Colin.

Bourdieu, P., et Passeron, J.-P. (1964). Les Héritiers. Les étudiants et la culture. Les éditions de minuit.

CDIP. (2019). Accord intercantonal sur la collaboration dans le domaine de la pédagogie spécialisée du 25 octobre 2007 (concordat sur la pédagogie spécialisée) : état d'avancement des procédures cantonales d'adhésion. Bern. https://edudoc.educa.ch/static/web/ arbeiten/sonderpaed/liste_rat_df.pdf

Cole, M. (2018). Education, Equality and Human Rights: Issues of Gender, 'Race', Sexuality, Disability and Social Class (4th Ed.). Routledge.

Collins, P., et Bilge, S. (2016). Intersectionality. Polity Press. 
Combet, B. (2013). Les effets primaires et secondaires de l'origine sociale lors du passage au gymnase en Suisse. Une comparaison de différentes méthodes de décomposition et de construction de la variable d'origine. Revue Suisse des Sciences de l'Éducation, 35(3), 447-472. https://doi.org/10.24452/sjer.35.3.4919

Confédération Suisse. (2002). Loi fédérale sur l'élimination des inégalités frappant les personnes handicapées. https://www.fedlex. admin.ch/eli/cc/2003/667/fr

Derouet, J.-L. (2004). Déconcentration, décentralisation et autonomie des établissements en France (1981-2003). D’un renouvellement des moyens de l'égalité au management en réseau. Revue Suisse des Sciences de l'Éducation, 26(1), 53-68. https://doi. org/10.24452/sjer.26.1.4670

Dozio, E. (2001). Évolution de la politique scolaire sur l'échec et l'inadaptation : le cas du Canton du Tessin. Revue Suisse des Sciences de l'Éducation, 23(2), 243-264. https://doi.org/10.24452/sjer.23.2.4605

Dubowy, M., Duzy, D., Pröscholdt, M., Schneider, W., Souvignier, E., et Gold, A. (2011). Que désigne «issu de l'immigration» à l'école enfantine ? Étude comparative de critères de classification et de leur relation avec les compétences linguistiques en allemand. Revue Suisse des Sciences de l'Éducation, 33(3), 355-376.

Dupont, V., et Lafontaine, D. (2011). Les choix d'études supérieures sont-ils liés à l'établissement secondaire fréquenté ? Revue Suisse des Sciences de l'Éducation, 33(3), 461-478. https://doi.org/10.24452/sjer.33.3.4869

Duru-Bellat, M. (1989). L'école des filles : Quelle formation pour quels rôles sociaux? L'Harmattan.

Duru-Bellat, M. (2001). Effets maîtres, effets établissements : quelle responsabilité pour l'école ? Revue Suisse des Sciences de l'Éducation, 23(2), 321-338. https://doi.org/10.24452/sjer.23.2.4608

Eckstein, B., Reusser, K., Stebler, R., et Mandel, D. (2013). Mise en œuvre de l'école intégrative : analyse de l'optimisme et des convictions des enseignants dans le canton de Zurich. Revue Suisse des Sciences de l'Éducation, 35(1), 91-112. https://doi. org/10.24452/sjer.35.1.4903

Eden, C. (2017). Gender, Education and Work. Inequalities and Intersectionality. Routledge.

Fassa, F., et Naef, C. (2015). Two interventions for equality in compulsary school : reasons for a contrasted becoming. Swiss Journal of Educational Research, 37(2), 267-284. https://doi.org/10.24452/sjer.37.2.4953

Genoud, P., Kappeler, G., et Guillod, M. (2015). Attitudes towards mathematics : are girls and boys equal in their approach to learning ? Swiss Journal of Educational Research, 37(2), 301-320. https://doi.org/10.24452/sjer.37.2.4955

Giudici, A., et Manz, K. (2018). Temps de l'éducation des garçons - temps de l'éducation des filles : égaux, de plus en plus égaux, inégaux ? Les différenciations de genres dans le savoir scolaire. Revue Suisse des Sciences de l'Éducation, 40(3), 603-620. https:// doi.org/10.24452/sjer.40.3.5119

Gremion, M., Noël, I., et Ogay, T. (2013). Éducation interculturelle et pédagogie spécialisée : tensions et ambiguïtés des discours sur la différence. Revue Suisse des Sciences de l'Éducation, 35(1), 53-70. https://doi.org/10.24452/sjer.35.1.4901

Griga, D., et Hadjar, A. (2013). Educational inequalities related to gender and migrant background at the transition to Higher Education. Findings from Switzerland, Germany and France. Swiss Journal of Educational Research, 35(3), 493-514. https:// doi.org/10.24452/sjer.35.3.4921

Gross, C., Gottburgsen, A., et Phoenix, A. (2016). Education systems and intersectionality. Dans A. Hadjar, et C. Gross (dir.), Education Systems and Inequalities: International Comparisons (p. 51-72). Policy Press.

Grossenbacher, S. (2000). Les femmes dans la formation professionnelle initiale et continue. Conséquences pour la question du genre dans la formation professionelle. Revue Suisse des Sciences de l'Éducation, 22(2), 295-309. https://doi.org/10.24452/ sjer.22.2.4568

Häfeli, K., Hättich, A., Schellenberg, C., et Schmaeh, N. (2015). Les raisons des différences croissantes dans la réussite professionnelle selon les genres. Revue Suisse des Sciences de l'Éducation, 37(2), 341-360. https://doi.org/10.24452/sjer.37.2.4957

Heikkinen, A. (2000). Biais liés au genre au niveau de la formation professionnelle dans les pays nordiques. Revue Suisse des Sciences de l'Éducation, 22(2), 357-376. https://doi.org/10.24452/sjer.22.2.4572

Hirschi, A. (2009). Une analyse typologique du marché suisse des places d'apprentissage : un désavantage structurel pour les jeunes femmes. Revue Suisse des Sciences de l'Éducation, 31(2), 317-334. https://doi.org/10.24452/sjer.31.2.5097

Imgrund, B. (2017). L’enseignement du français langue étrangère à l'école primaire. Développement de compétences dans un contexte multiculturel. Revue Suisse des Sciences de l'Éducation, 39(1), 171-190. https://doi.org/10.24452/sjer.39.1.5005

Kost, J. (2013). Perméabilité et accès aux études supérieures en Suisse. Revue Suisse des Sciences de l'Éducation, 35(3), 473-492. https://doi.org/10.24452/sjer.35.3.4920

Kronberger, S., Oberlechner, M., et Bramberger, A. (dir.). (2017). Bildung - Intersektionalität - Geschlecht. StudienVerlag.

Kuyumcu, R., et Senyldiz, A. (2011). Les expériences littérales familiales d'enfants préscolaires turcophones et russophones. Revue Suisse des Sciences de l'Éducation, 33(1), 109-124. https://doi.org/10.24452/sjer.33.1.4852

Labudde, P. (2010). Analyser, modéliser et repenser l'enseignement des sciences naturelles. Revue Suisse des Sciences de l'Éducation, 32(3), 371-392. https://doi.org/10.24452/sjer.32.3.4840

Lasne, A. (2017). Obstacles à la fondation d'une relation de coéducation entre parents et enseignants. Revue Suisse des Sciences de l'Éducation, 39(2), 291-306. https://doi.org/10.24452/sjer.39.2.5011

Lavoie, G., Thomazet, S., Feuilladieu, S., Pelgrims, G., et Ebersold, S. (2013). Construction sociale de la désignation des élèves à «besoins éducatifs particuliers» : incidences sur leur scolarisation et sur la formation des enseignants. ALTER, European Journal of Disability Research, 7(2), 93-101.

Leemann, R. (2002). Inégalité des chances dans l'accès à une carrière scientifique. Revue Suisse des Sciences de l'Éducation, 24(2), 197-222. https://doi.org/10.24452/sjer.24.2.4627

Leutwyler, B., Steinger, E., et Sieber, P. (2009). Un modèle par étape de réflexions sur la normalité. Les différentes positions des enseignant-e-s en matière de réflexion sur l'hétérogénéité à l'école et dans l'enseignement. Revue Suisse des Sciences de l'Éducation, 31(3), 565-584. https://doi.org/10.24452/sjer.31.3.4819

Levitt, P., et Jaworsky, B. N. (2007). Transnational migration studies: Past developments and future trends. Annual Review of Sociology, 33, 129-156. 
Lind, I., et Löther, A. (2007). Les chances des femmes en science : une question de culture disciplinaire ? Analyses rétrospectives de la qualification et résultats actuels de la recherche. Revue Suisse des Sciences de l'Éducation, 29(2), 249-272. https://doi. org/10.24452/sjer.29.2.4772

Mazzella, S. (2014). Sociologie des migrations. PUF.

Mitchell, D., Simmons, C., et Greyerbiehl, L. (2014). Intersectionality et Higher Education: Theory, Research, et Praxis. Peter Lang.

Mosconi, N. (1998). Égalité des sexes en éducation et formation. Presses universitaires de France.

Mosconi, N. (2000). La formation professionnelle des femmes en France. Revue Suisse des Sciences de l'Éducation, 22(2), 343-355. https://doi.org/10.24452/sjer.22.2.4571

Müller, R. (2001). La situation des jeunes de langue étrangère au degré secondaire II - Intégration ou discrimination ? Revue Suisse des Sciences de l'Éducation, 23(2), 265-298. https://doi.org/10.24452/sjer.23.2.4606

Neuenschwander, M. P. (2007). Le passage au secondaire II : problèmes, constats, solutions. Dans Aspects de la transition «secondaire I - secondaire II", CDIP. www.nahtstelle-transition.ch/files/nst7131.pdf

Neumann, U. (2007). Schools in transnational communities. The "Förmig-Program" to the promotion of children and youngsters with a migration background. Swiss Journal of Educational Research, 29(3), 435-452. https://doi.org/10.24452/sjer.29.3.4580

Noël, I., Gremaud, J., et Hofstetter, D. (2019). Quand les velléités d'intégration révèlent les éternels exclus : analyse de textes législatifs et règlementaires fribourgeois de 1900 à nos jours. Revue Suisse des Sciences de l'Éducation, 41(3), 708-724. https:// bop.unibe.ch/sjer/article/view/6024

Oertel, L., Schmidt, M., et Melzer, W. (2009). La violence à l'école - Les relations entre l'hétérogénéité de la classe et les expériences de harcèlement moral. Revue Suisse des Sciences de l'Éducation, 36(1), 99-116. https://doi.org/10.24452/sjer.36.1.4810

ONU. (2006). Convention relative aux droits des personnes handicapées (CDPH). New York.

Parini, L. (2010). Le concept de genre : constitution d'un champ d'analyse, controverses épistémologiques, linguistiques et politiques. Socio-Logos, 5. https://doi.org/10.4000/socio-logos.2468

Pavard, B. (2018). Faire naître et mourir les vagues : comment s'écrit l'histoire des féminismes. Itinéraires. Littérature, Textes, Cultures, 2017(2). http://journals.openedition.org/itineraires/3787

Pelt, V., et Poncelet, D. (2011). Une recherche-action. Connaître, accompagner et provoquer le changement en sciences de l'éducation. Revue Suisse des Sciences de l'Éducation, 33(3), 495-510.

Perregaux, C. (2007). Les contextes pluriculturels et plurilingues, lieux de transformation des connaissances et des rapports sociaux dans l'école ? Revue Suisse des Sciences de l'Éducation, 29(3), 417-435. https://doi.org/10.24452/sjer.29.3.4579

Pons, F., Doudin, P.-A., et Pini, G. (2000). Identité culturelle et scolarité d'élèves portugais en Suisse. Revue Suisse des Sciences de l'Éducation, 22(3), 585-606. https://doi.org/10.24452/sjer.22.3.4592

Pool Maag, S. (2016). Les défis de la transition de l'école à la profession : les résultats d'une recherche sur l'intégration professionnelle des jeunes handicapés en Suisse. Revue Suisse des Sciences de l'Éducation, 38(3), 591-610. https://doi.org/10.24452/ sjer.38.3.4995

Preite, L. (2018). La culture « en ligne " des jeunes considérés comme étant «à risques ». Revue Suisse des Sciences de l'Éducation, 40(2), 335-350. https://doi.org/10.24452/sjer.40.2.5064

Prengel, A. (2007). In a «floating state»: Schools and transgressive communities. Swiss Journal of Educational Research, 29(3), 363-378. https://doi.org/10.24452/sjer.29.3.4576

Prost, A. (2001). La démocratisation de l'enseignement en France depuis la Seconde Guerre mondiale. Revue Suisse des Sciences de l'Éducation, 23(1), 73-94. https://doi.org/10.24452/sjer.23.1.4597

Prud'homme, L., Duchesne, H., Bonvin, P., et Vienneau, R. (dir.). (2016). Linclusion scolaire : ses fondements, ses acteurs et ses pratiques. De Boeck Supérieur.

Quennerstedt, A. (2011). The construction of children's rights in education - a research synthesis. International Journal of Children's Rights, 19, 661-678.

Quennerstedt, A., et Moody, Z. (2020). Educational children's rights research 1989-2019: achievements, gaps and future prospects. International Journal of Children's Rights, 28, 183-208.

Quesel, C., Möser, G., et Husfeldt, V. (2014). Impact des différences sociales sur les climats relationnel, éducatif et organisationnel dans l'école obligatoire en Suisse. Revue Suisse des Sciences de l'Éducation, 36(2), 283-306. https://doi.org/10.24452/ sjer.36.2.4937

Rochex, J.-Y. (2001). Échec scolaire et démocratisation : enjeux, réalités, concepts, problématiques et résultats de recherche. Revue Suisse des Sciences de l'Éducation, 23(2), 339-356. https://doi.org/10.24452/sjer.23.2.4609

Rousseau, N., Bergeron, G., et Vienneau, R. (2013). L'inclusion scolaire pour gérer la diversité : des aspects théoriques aux pratiques dites efficaces. Revue Suisse des Sciences de l'Éducation, 35(1), 71-90. https://doi.org/10.24452/sjer.35.1.4902

Rousseau, N., Point, M., Vienneau, R., Desmarais, M.-E., et Desmarais, K. (2017). Les apports et les limites liés aux pratiques inclusives et la place de la collaboration dans ces pratiques : une métasynthèse. Revue Suisse des Sciences de l'Éducation, 39(1), 21-40. https://doi.org/10.24452/sjer.39.1.4997

Ruşitoru, M.-V. (2017). Léducation à l'épreuve des flux migratoires dans les destinations de langue française. La situation des enfants sans-papiers en France et au Québec. Revue Suisse des Sciences de l'Éducation, 39(1), 41-56. https://doi.org/10.24452/ sjer.39.1.4998

Sánchez Abchi, V. (2015). Développement de capacités narratives des enfants bilingues : l'acquisition de l'espagnol dans le contexte suisse-allemand. Revue Suisse des Sciences de l'Éducation, 37(1), 21-38. https://doi.org/10.24452/sjer.37.1.4941

Soilemezi, D., et Linceviciute, S. (2018). Synthesizing qualitative research: Reflections and lessons learnt by two new reviewers. International Journal of Qualitative Methods, 17, 1-14.

Schärer, M. E. (2000). Femmes et formation professionnelle continue dans une perspective historique. Le cas de Genève : 1880-1914. Schweizerische Zeitschrift für Bildungswissenschaften, 22(2), 311-330. https://doi.org/10.24452/sjer.22.2.4570

Stecher, L. (2007). Garçons et filles à l'école à plein temps. Revue Suisse des Sciences de l'Éducation, 29(2), 209-230. https://doi. org/10.24452/sjer.29.2.4770 
Sutcliffe, K., Oliver, S., et Richardson, K. (2012). Describing and analysing studies. Dans D. Gough, S. Oliver, et J. Thomas (dir.), An Introduction to Systematic Reviews (p. 123-144). Sage.

Teddlie, C., et Tashakkori, A. (2003). Major issues and controversies in the use of mixed methods in the social and behavioral sciences. Dans A. Tashakkori, et C. Teddlie (dir.), Handbook of Mixed Methods in Social and Behavioral Research (p. 3-50). SAGE Publications.

Tenorth, H.-E. (2008). Asservissement et persévérance - L'École face aux conditions des dictatures allemandes. Résultats et aspects de l'analyse. Revue Suisse des Sciences de l'Éducation, 30(2), 275-298. https://doi.org/10.24452/sjer.30.2.4794

UNESCO. (1994). Déclaration de Salamanque et cadre d'action pour l'éducation et les besoins spéciaux. Salamanque. https://unesdoc. unesco.org/ark :/48223/pf0000098427_fre

Unwin, L. (2000). Formation professionnelle, préparation et entrée dans le monde du travail des jeunes en Angleterre et au Pays de Galles : voyage à travers un passage marqué par des spécificités liées au genre. Revue Suisse des Sciences de l'Éducation, 22(2), 331-342. https://doi.org/10.24452/sjer.22.2.4569

Winzer, M., et Mazurek, K. (2017). Diversity, difference, and disability: Conceptual contradictions and present practice in inclusive schooling for students with disabilities. International Dialogue in Education, 1(225).

Mots-clés : Égalité ; diversité ; genre ; migration ; classe sociale ; inclusion ; synthèse de recherche

\section{Vielfalt und Gleichstellung im Bildungsbereich (2000-2019): Entwicklungen, Herausforderungen und Perspektiven}

Zusammenfassung

Angesichts der Vielfalt des Publikums im Bildungsbereich bildet das Thema Chancengleichheit einen Schwerpunkt in der Bildungsforschung. Die Ungleichheiten sowie die Schwierigkeit, eine Balance zwischen individuellen Bedürfnissen und dem Aspekt der Nicht-Diskriminierung zu finden, bestehen fort. So ist nicht verwunderlich, dass diese im Zentrum der Debatten stehen, in denen versucht wird, die theoretischen und praktischen Herausforderungen der Vielfalt im Bildungsbereich zu beleuchten. Dieser Beitrag bietet einen systematischen Überblick über das Forschungsfeld, das sich mit Fragen der Vielfalt und Gleichstellung im Bildungsbereich befasst, wie es in den letzten zwanzig Jahren (2000-2019) in der wichtigsten erziehungswissenschaftlichen Zeitschrift in der Schweiz skizziert wurde. Ausgehend von der Beschreibung der Themen, an denen die Bildungsforschenden arbeiten, werden Entwicklungen, Zäsuren und Kontinuitäten sowie die wichtigsten Themen identifiziert.

Schlagworte: Gleichheit; Vielfalt; Gender; Migration; Soziale Klasse; Inklusion; Forschungssynthese

\section{Diversità e uguaglianza nell'istruzione (2000-2019): sviluppi, sfide e prospettive}

\section{Riassunto}

Di fronte alla diversità del pubblico scolastico, l'uguaglianza è un tema di crescente attenzione nella ricerca in campo educativo. Le persistenti disuguaglianze e il difficile equilibrio tra l'integrazione delle esigenze individuali e la non discriminazione sono al centro dei dibattiti, nel tentativo di mettere in luce le sfide teoriche e pratiche sulla diversità in ambito pedagogico e didattico. Questo contributo propone una rassegna sistematica del campo di ricerca che si occupa di questioni legate alla diversità e all'uguaglianza nell'istruzione, come delineato degli ultimi vent'anni (2000-2019) nella principale rivista del settore delle scienze dell'educazione in Svizzera. Gli sviluppi, i punti di rottura e la continuità così come le tematiche trasversali più significative sono di seguito evidenziati, a partire dalla descrizione delle questioni sulle quali lavorano le ricercatrici e i ricercatori in scienze dell'educazione.

Parole chiave: Uguaglianza; Diversità; Genere; Migrazione; Classe sociale; Inclusione; Sintesi della ricerca 


\section{Diversity and equality in education (2000-2019): developments, challenges and prospects}

Summary

With the diversity of school audiences, equality is an increasing subject of attention in educational research. Persisting inequalities and the difficult balance between individual needs and non-discrimination are at the heart of debates, in an attempt to highlight the theoretical and practical challenges of diversity in education. This contribution proposes a systematic review of the research field dealing with issues of diversity and equality in education, as outlined over the last twenty years (2000-2019) in the main educational sciences review in Switzerland. Developments, breaking points and continuities are identified, as well as the most significant cross-cutting themes, based on the description of issues on which educational researchers are working.

Keywords: Equality; diversity; gender; migration; social class; inclusion; research synthesis

Zoe Moody est professeure à la Haute école pédagogique du Valais et collaboratrice scientifique à la cellule Inter- et transdisciplinarité du Centre interfacultaire en Droits de l'enfant de l'Université de Genève. Ses travaux de recherche portent sur les droits de l'enfant en contextes éducatifs, le vivre ensemble et la diversité à l'école, les discriminations et les violences scolaires (school bullying).

Haute école pédagogique du Valais, Route du Simplon 13, CH-1890 St-Maurice, Valais

E-Mail : zoe.moody@hepvs.ch

Isabel Valarino, collaboratrice de recherche au Service de la recherche en éducation (SRED), travaille actuellement sur la pédagogie spécialisée et les parcours scolaires des mineur·e·s placé.e.s.

Service de la recherche en éducation, Département de l'instruction publique, de la formation et de la jeunesse (DIP), République et canton de Genève, 12, quai du Rhône, CH-1205 Genève

E-Mail : isabel.valarino@etat.ge.ch 